\title{
SUPPLEMENT TO: BAYESIAN LATENT PATTERN MIXTURE MODELS FOR HANDLING ATTRITION IN PANEL STUDIES WITH REFRESHMENT SAMPLES
}

\author{
By Yajuan Si* , Jerome P. Reiter ${ }^{\dagger}$ and D. Sunshine Hillygus ${ }^{\dagger}$ \\ University of Wisconsin-Madison* and Duke University ${ }^{\dagger}$
}

This online supplement comprises five sections. Section 1 and Section 2 present the Markov chain Monte Carlo algorithms for a Dirichlet process mixture of products of multinomial (DPMPM) model and for the Bayesian latent pattern mixture (BLPM) model. The former model imposes conditional independence between $\left(X, Y_{2}\right)$ and $W$ within classes. Section 3 presents the analyses of the AP Yahoo News (APYN) election panel data when using the DPMPM model rather than BLPM model. Section 4 presents results of an analysis of the APYN data using the semi-parametric additive non-ignorable model of Si et al. (2014), including corresponding posterior predictive checks. Section 5 includes the posterior predictive checks for the BLPM model. Throughout we use the same notation as in the main text.

1. Posterior Computation Algorithm for DPMPM. Here we outline the MCMC algorithm for the DPMPM model, which was used for comparisons with the BLPM in Section 6 of the main text.

Step 1: For $i=1, \ldots, N$, sample $s_{i} \in\{1, \ldots, K\}$ from a multinomial distribution with one trial and probabilities

$$
\operatorname{Pr}\left(s_{i}=h \mid-\right)=\frac{\pi_{h} \rho_{h}^{W_{i}}\left(1-\rho_{h}\right)^{1-W_{i}} \prod_{j=1}^{q} \psi_{h j Z_{i j}}}{\sum_{k=1}^{K} \pi_{k} \rho_{k}^{W_{i}}\left(1-\rho_{k}\right)^{1-W_{i}} \prod_{j=1}^{q} \psi_{k j Z_{i j}}} .
$$

Step 2: For $h=1, \ldots, K-1$, sample $V_{h}$ from the Beta distribution

$$
\left(V_{h} \mid-\right) \sim \operatorname{Beta}\left(1+n_{h}, \alpha+\sum_{k=h+1}^{K} n_{k}\right),
$$

where $n_{h}=\sum_{i=1}^{N} I\left(s_{i}=h\right)$. Compute $\pi_{h}$ from $\pi_{h}=V_{h} \prod_{k<h}\left(1-V_{k}\right)$ with $V_{K}=1$.

Step 3a: For $h=1, \ldots, K$ and $j=1, \ldots, q$, sample $\psi_{h j}=\left(\psi_{h j 1}, \ldots, \psi_{h j d_{j}}\right)$ from the Dirichlet distribution,

$$
\left(\psi_{h j} \mid-\right) \sim \operatorname{Dirichlet}\left(1+\sum_{i: s_{i}=h} I\left(Z_{i j}=1\right), \ldots, 1+\sum_{i: s_{i}=h} I\left(Z_{i j}=d_{j}\right)\right) .
$$


Step 3b: For $h=1, \ldots, K$, sample $\rho_{h}$ from the Beta distribution,

$$
\left(\rho_{h} \mid-\right) \sim \operatorname{Beta}\left(1+\sum_{i: s_{i}=h} I\left(W_{i}=1\right), 1+\sum_{i: s_{i}=h} I\left(W_{i}=0\right)\right) .
$$

Step 4: Sample $\alpha$ from the Gamma distribution,

$$
(\alpha \mid-) \sim \operatorname{Gamma}\left(a_{\alpha}+K-1, b_{\alpha}-\log \pi_{K}\right) .
$$

Step 5: For each $Z_{i, j}$ that is missing in the collected data, sample a new value from the multinomial distribution,

$$
\left(Z_{i, j} \mid-\right) \sim \operatorname{Multinomial}\left(\left\{1, \ldots, d_{j}\right\}, \psi_{s_{i} j}, \ldots, \psi_{s_{i} j d_{j}}\right) .
$$

Step 6: Sample values of $W_{i}$ for units in the refreshment sample from the Bernoulli distribution,

$$
\left(W_{i} \mid-\right) \sim \operatorname{Bernoulli}\left(\rho_{s_{i}}\right) .
$$

2. Posterior Computation Algorithm for BLPM. Here we outline the MCMC algorithm for the BLPM model described in Section 4 of the main text.

Step 1: For $i=1, \ldots, N$, sample $s_{i} \in\{1, \ldots, K\}$ from a multinomial distribution with one trial and probability $\operatorname{Pr}\left(s_{i}=h \mid-\right)$ equal to

$$
\frac{\pi_{h} \rho_{h}^{W_{i}}\left(1-\rho_{h}\right)^{1-W_{i}} \prod_{j=1}^{q_{0}} \psi_{h j Z_{i j}}^{\left(W_{i}\right)} \prod_{j=q_{0}+1}^{q_{0}+q_{1}} \psi_{h j Z_{i j}} \prod_{j=q_{0}+q_{1}+1}^{q} \psi_{h j Z_{i j}}^{\left(W_{i}\right)}}{\sum_{k=1}^{K} \pi_{k} \rho_{k}^{W_{i}}\left(1-\rho_{k}\right)^{1-W_{i}} \prod_{j=1}^{q_{0}} \psi_{k j}^{\left(W_{i}\right)} \prod_{j=q_{0}+1}^{q_{0}+q_{1}} \psi_{k j} Z_{i j} \prod_{j=q_{0}+q_{1}+1}^{q} \psi_{k j Z_{i j}}^{\left(W_{i}\right)}} .
$$

Step 2: For $h=1, \ldots, K-1$, sample $V_{h}$ from the Beta distribution,

$$
\left(V_{h} \mid-\right) \sim \operatorname{Beta}\left(1+n_{h}, \alpha+\sum_{k=h+1}^{K} n_{k}\right)
$$

where $n_{h}=\sum_{i=1}^{N} I\left(s_{i}=h\right)$. Compute $\pi_{h}$ from $\pi_{h}=V_{h} \prod_{k<h}\left(1-V_{k}\right)$ with $V_{K}=1$.

Step 3a: For $h=1, \ldots, K, j \in\left\{1, \ldots q_{0}, q_{0}+q_{1}+1, \ldots, q\right\}$, sample $\psi_{h j}^{(1)}=\left(\psi_{h j 1}^{(1)}, \ldots, \psi_{h j d_{j}}^{(1)}\right)$ from the Dirichlet distribution,

$$
\left(\psi_{h j}^{(1)} \mid-\right) \sim \operatorname{Dirichlet}\left(1+\sum_{i: s_{i}=h, w_{i}=1} I\left(Z_{i j}=1\right), \ldots, 1+\sum_{i: s_{i}=h, w_{i}=1} I\left(Z_{i j}=d_{j}\right)\right) .
$$


Step 3b: For $h=1, \ldots, K, j \in\left\{1, \ldots q_{0}, q_{0}+q_{1}+1, \ldots, q\right\}$, sample $\psi_{h j}^{(0)}=\left(\psi_{h j 1}^{(0)}, \ldots, \psi_{h j d_{j}}^{(0)}\right)$ from the Dirichlet distribution,

$$
\left(\psi_{h j}^{(0)} \mid-\right) \sim \operatorname{Dirichlet}\left(1+\sum_{i: s_{i}=h, w_{i}=0} I\left(Z_{i j}=1\right), \ldots, 1+\sum_{i: s_{i}=h, w_{i}=0} I\left(Z_{i j}=d_{j}\right)\right) .
$$

Step 3c: For $h=1, \ldots, K, j \in\left\{q_{0}+1, \ldots, q_{0}+q_{1}\right\}$, sample $\psi_{h j}=\left(\psi_{h j 1}, \ldots, \psi_{h j d_{j}}\right)$ from the Dirichlet distribution,

$$
\left(\psi_{h j} \mid-\right) \sim \operatorname{Dirichlet}\left(1+\sum_{i: s_{i}=h} I\left(Z_{i j}=1\right), \ldots, 1+\sum_{i: s_{i}=h} I\left(Z_{i j}=d_{j}\right)\right) .
$$

Step 3d: For $h=1, \ldots, K$, sample $\rho_{h}$ from the Beta distribution,

$$
\left(\rho_{h} \mid-\right) \sim \operatorname{Beta}\left(1+\sum_{i: s_{i}=h} I\left(W_{i}=1\right), 1+\sum_{i: s_{i}=h} I\left(W_{i}=0\right)\right) .
$$

Step 4: Sample $\alpha$ from the Gamma distribution,

$$
(\alpha \mid-) \sim \operatorname{Gamma}\left(a_{\alpha}+K-1, b_{\alpha}-\log \pi_{K}\right) .
$$

Step 5a: For $j \in\left\{1, \ldots q_{0}, q_{0}+q_{1}+1, \ldots, q\right\}$, for each $Z_{i j}$ that is missing in the collected data in the panel or refreshment sample, sample a new value from the multinomial distribution,

$$
\left(Z_{i, j} \mid-\right) \sim \operatorname{Multinomial}\left(\left\{1, \ldots, d_{j}\right\}, \psi_{s_{i} j 1}^{\left(w_{i}\right)}, \ldots, \psi_{s_{i} j d_{j}}^{\left(w_{i}\right)}\right) .
$$

Step 5b: For $j \in\left\{q_{0}+1, \ldots, q_{0}+q_{1}\right\}$, for each $Z_{i j}$ that is missing in the collected data in the panel or refreshment sample, sample a new value from the multinomial distribution,

$$
\left(Z_{i, j} \mid-\right) \sim \operatorname{Multinomial}\left(\left\{1, \ldots, d_{j}\right\}, \psi_{s_{i} j 1}, \ldots, \psi_{s_{i} j d_{j}}\right) .
$$

Step 6: For all individuals in the refreshment sample, sample $W_{m i s}$ from the Bernoulli distribution,

$$
\left(W_{i} \mid-\right) \sim \operatorname{Bernoulli}\left(\operatorname{Pr}\left(W_{i}=1 \mid Z_{i}, s_{i}, \Psi\right)\right),
$$

where $\operatorname{Pr}\left(W_{i}=1 \mid-\right)$ is equal to

$$
\frac{f\left(X_{i} \mid s_{i}, W_{i}=1, \Psi\right) f\left(Y_{i, 2} \mid s_{i}, W_{i}=1, \Psi\right) \operatorname{Pr}\left(W_{i}=1 \mid s_{i}, \Psi\right)}{\left.\sum_{w \in\{0,1\}} f\left(X_{i} \mid s_{i}, W_{i}=w, \Psi\right) f\left(Y_{i, 2} \mid s_{i}, W_{i}=w, \Psi\right)\right) \operatorname{Pr}\left(W_{i}=w \mid s_{i}, \Psi\right)} .
$$


3. Results of DPMPM on APYN Data. The main text uses the BLPM for analysis of the APYN data. Here, we present some results using the DPMPM model. We set the initial values of parameters following similar strategies as those in Section 6.2. We run the MCMC chain for 150,000 iterations, collecting $m=50$ imputed datasets after thinning. We implement some of the same analyses performed in Section 6.3.

The DPMPM results in substantially different conclusions than those based on the BLPM, suggesting no bias due to attrition. The marginal estimate for Obama favorability for panel non-attriters in the panel is 0.551, with $95 \%$ confidence interval $(0.527,0.575)$. The same estimate computed using only the panel attriters is 0.558 with $95 \%$ confidence interval $(0.509$, 0.618). Figure 1 displays the estimates of Obama favorability for the subgroups in Figure 3 in the main article. The completed panel estimates are similar to those for the non-attriters, so that the DPMPM does not appear to leverage the information in the refreshment sample. Apparently, the conditional independence assumption for $Y_{2}$ is inappropriate for these data.

We also estimated the DPMPM model without controlling for $W$, which effectively enforces a missing at random assumption. The results, not shown here for brevity, are very similar to those in Figure 1.

4. Results of semi-parametric additive nonignorable model on APYN data. As described in Section 6.3 and 6.4 of the main text, we analyzed the APYN data using the semi-parametric additive non-ignorable (AN) model of Si et al. (2014), including corresponding posterior predictive checks. We set the initial values of parameters following similar strategies as those in Section 6.2 and run the MCMC chain for 100,000 iterations. We create $m=50$ completed datasets that we use for the analyses described in Section 6.3.

Like the BLPM, the semi-parametric AN model also indicates that attrition is non-ignorable. The estimated coefficient for $Y_{2}$ in the selection model for $W$ is significantly different from zero. Comparing the substantive analyses done for the BLPM in Figure 3 and 4 of the main text, we find that the semi-parametric AN model yields similar results. Figure 2 displays estimated probabilities for Obama favorability for each of the sub-groups defined by the time-invariant variables. Like the BLPM, the semi-parametric AN model appears to correct for attrition bias. The point estimates for BLPM and the semi-parametric AN model differ a bit; however, the large variances after multiple imputation make the comparison practically indistinguishable.

We also examined posterior predictive probabilities, as noted in Section 6.4 of the main text. Figure 3 displays a histogram of the 38 values of $p p p$ 


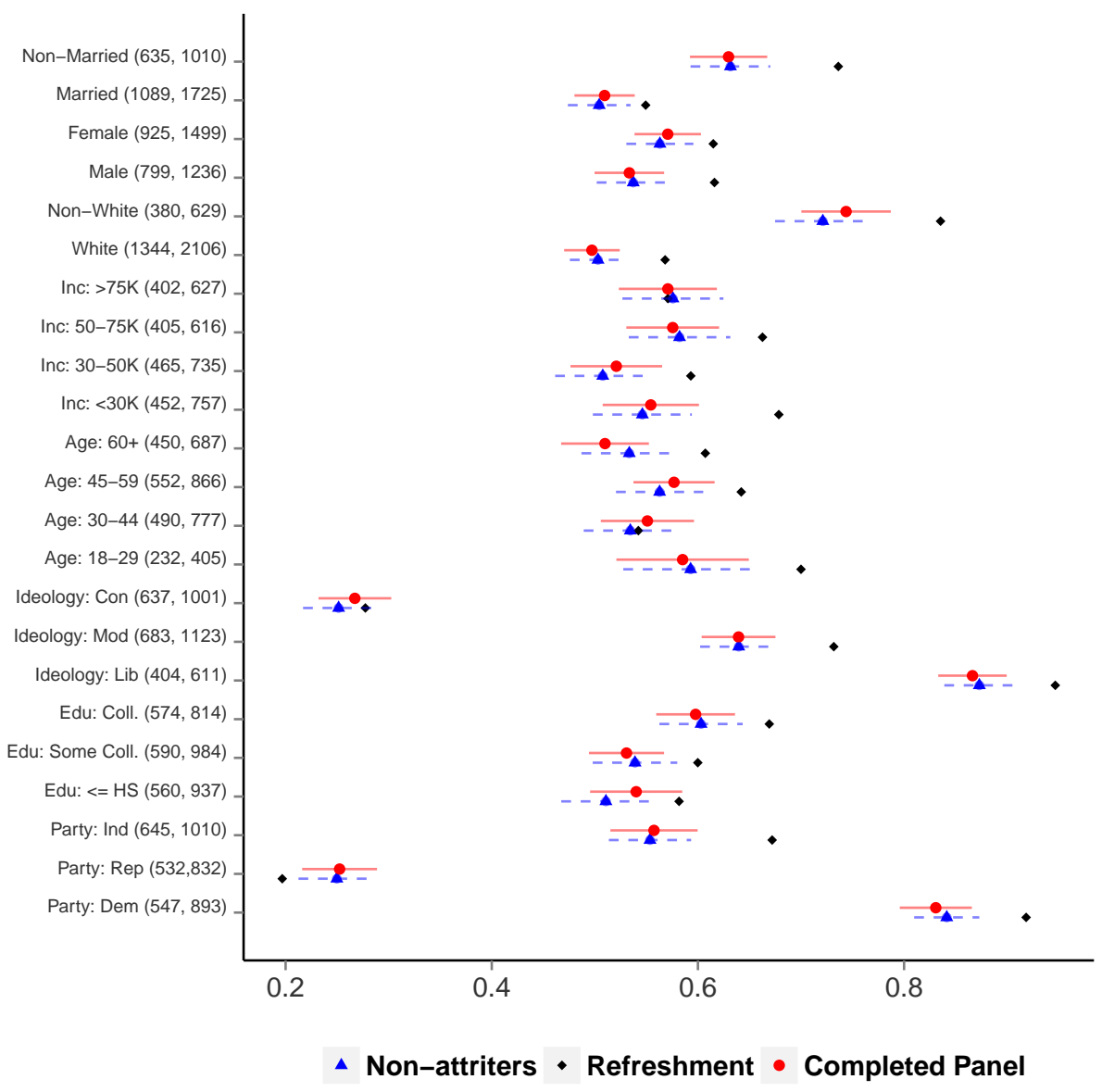

FIG 1. Point estimates and 95\% confidence intervals for Obama favorability in various sub-groups. Results presented for the $N_{c p}$ panel non-attriters, the $N_{r}$ refreshment sample cases, and the $N_{p}$ panel participants. Inferences based on unweighted analyses of the $m=50$ completed datasets, after multiple imputation of missing values due to item nonresponse and attrition via the DPMPM model including $W$. The numbers in parentheses are the corresponding subgroup sizes, the first being the size among non-attriters and the second being among the completed panel. We randomly select one imputed dataset to obtain the sample sizes when the background variables are subject to item nonresponse.

based on the semi-parametric AN model. Many $p p p$ values are quite small, suggesting that overall the semi-parametric AN model does not to fit the APYN data particularly well, especially compared to the BLPM. 

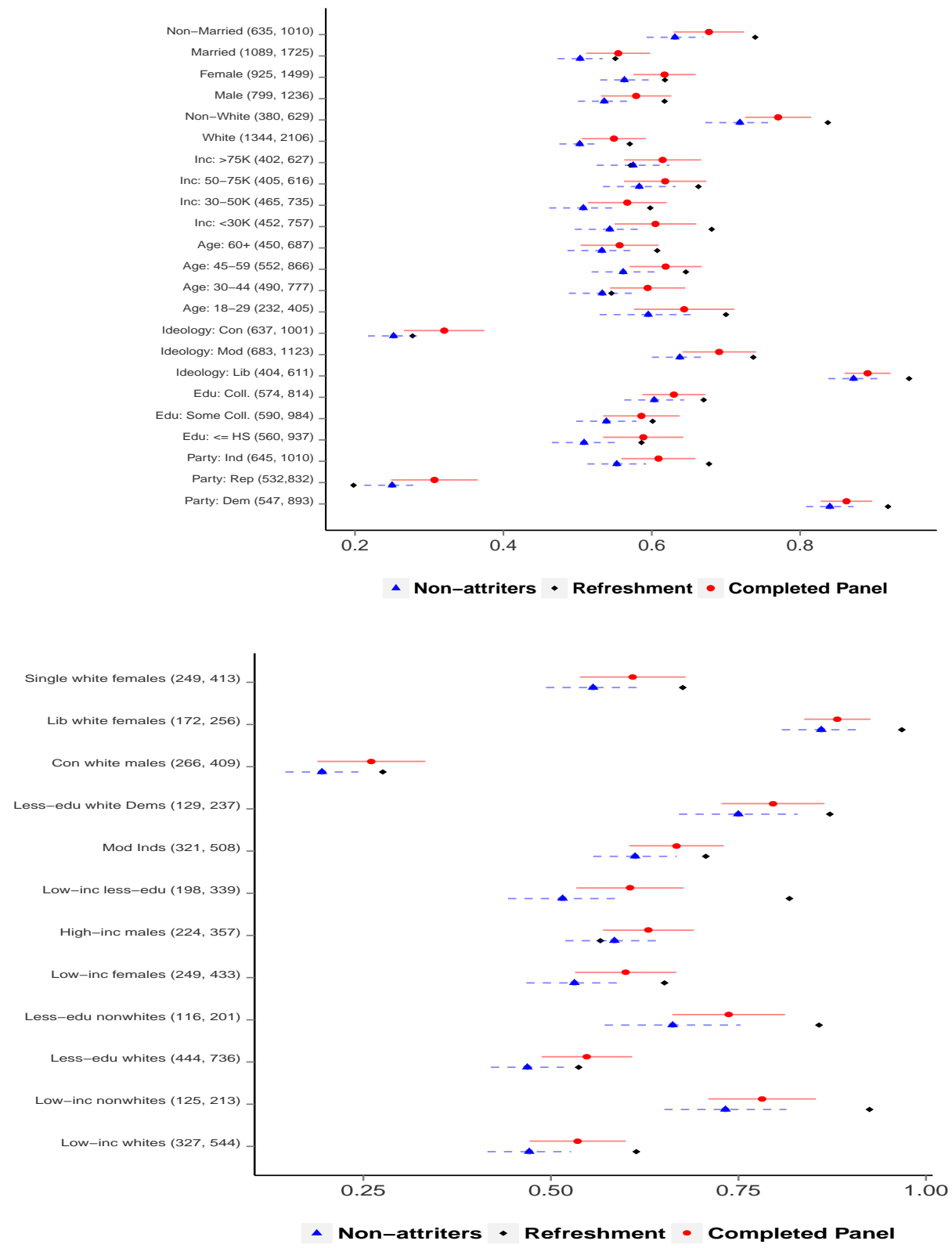

FIG 2. Point estimates and 95\% confidence intervals for Obama favorability in sub-groups. Results presented for the $N_{c p}$ panel non-attriters, the $N_{r}$ refreshment samples, and the $N_{p}$ panel participants. Inferences based on unweighted analyses of the $m=50$ completed datasets, after multiple imputation of missing values via the AN model. The numbers in parentheses are the corresponding subgroup sizes, the first being the size among nonattriters and the second being among the completed panel. We randomly select one imputed dataset to obtain the sample sizes when the background variables are subject to item nonresponse. 


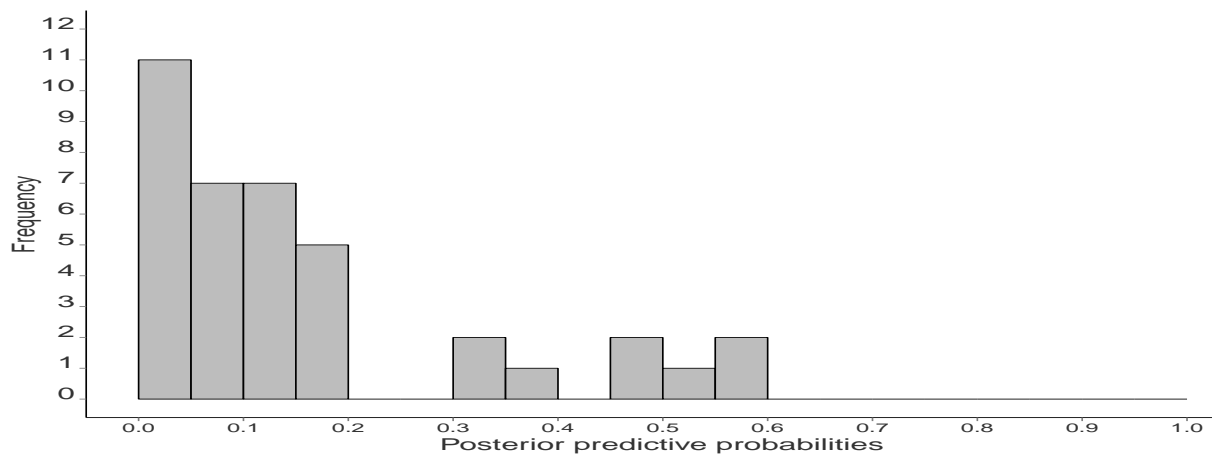

FIG 3. Histogram of the 38 ppp values from the posterior predictive checks of the APYN analyses with AN model.

5. Frequency distribution for the posterior predictive probabilities for BLPM model. Figure 4 displays a histogram of the 38 values of $p p p$ with BLPM, as described in Section 6.4 of the main text. None of $p p p$ values are below 0.20 , suggesting no evidence of serious lack of model fit.

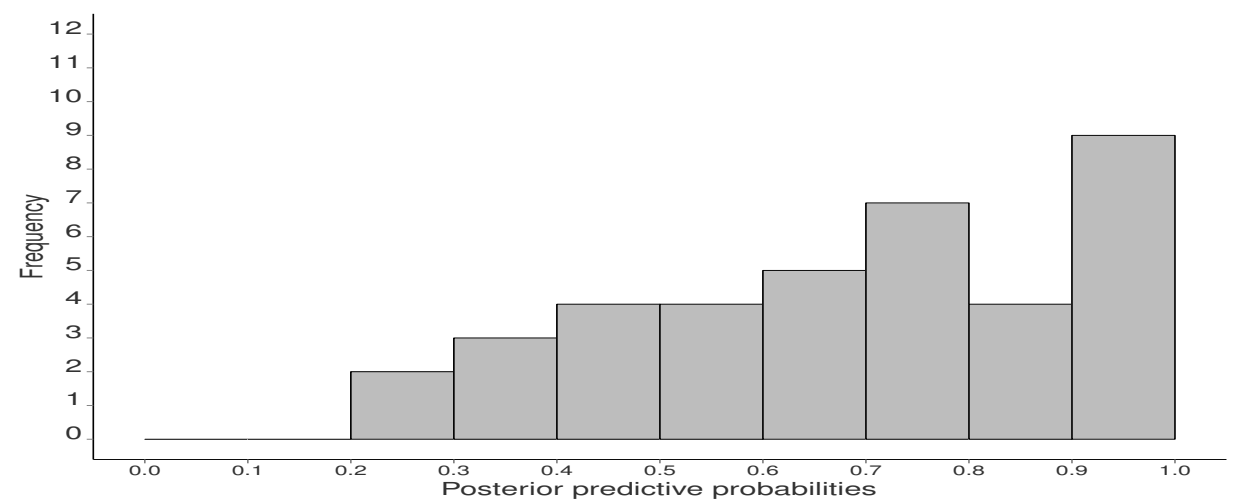

FIG 4. Histogram of the 38 ppp values from the posterior predictive checks of the APYN analyses with BLPM model.

\section{References.}

Si, Y., J. P. Reiter, and D. S. Hillygus (2014). Semi-parametric selection models for potentially non-ignorable attrition in panel study with refreshment sample. Political Analysis 23, 92-112. 
Department of Biostatistics \& Medical Informatics Department of Statistical Science Department of Population Health Sciences Duke University

UNIVERSITY OF WISCONSIN-MADISON

MADISON, WI 53726

DURHAM, NC 27708

E-MAIL: ysi@biostat.wisc.edu

E-MAIL: jerry@stat.duke.edu

Department of Political Science

DUKE UNIVERSITY

DURHAM, NC 27708

E-MAIL: hillygus@duke.edu 